\title{
Tenofovir or zidovudine in second-line antiretroviral therapy after stavudine failure in southern Africa
}

Gilles Wandeler, Florian Gerber, Julia Rohr, Benjamin H Chi, Catherine Orrell, Cleophas Chimbetete, Hans Prozesky, Andrew Boulle, Christopher J Hoffmann, Thomas Gsponer, Matthew P Fox, Marcel Zwahlen, Matthias Egger, leDEA Southern Africa

Antiviral Therapy 2013; 10.3851/IMP2710

Submission date 16th October 2013

Acceptance date 4th November 2013

Publication date 3rd December 2013

This provisional PDF matches the article and figures as they appeared upon acceptance.

Copyedited and fully formatted PDF and full text (HTML) versions will be made available soon.

For information about publishing your article in Antiviral Therapy go to http://www.intmedpress.com/index.cfm?pid=12 


\section{Short communication}

\section{Tenofovir or zidovudine in second-line antiretroviral therapy after stavudine failure in southern Africa}

Gilles Wandeler ${ }^{1,2,3 *}$, Florian Gerber ${ }^{1}$, Julia Rohr ${ }^{4}$, Benjamin H Chi ${ }^{5}$, Catherine Orrell ${ }^{6}$, Cleophas Chimbetete ${ }^{7}$, Hans Prozesky ${ }^{8}$, Andrew Boulle ${ }^{9}$, Christopher J Hoffmann ${ }^{10,11}$, Thomas Gsponer ${ }^{1}$, Matthew P Fox ${ }^{4,12}$, Marcel Zwahlen ${ }^{1}$, Matthias Egger ${ }^{1}$, leDEA Southern Africa

${ }^{1}$ Institute of Social and Preventive Medicine (ISPM), University of Bern, Bern, Switzerland

${ }^{2}$ Department of Infectious Diseases, Bern University Hospital and University of Bern, Bern, Switzerland,

${ }^{3}$ Department of Infectious Diseases, University of Dakar, Dakar, Senegal

${ }^{4}$ Boston University Center for Global Health and Development, Boston, MA, USA

${ }^{5}$ Centre for Infectious Disease Research in Zambia, Lusaka, Zambia

${ }^{6}$ The Desmond Tutu HIV Centre, Institute of Infectious Disease and Molecular Medicine, University of Cape Town, Cape Town, South Africa

${ }^{7}$ Newlands Clinic, Harare, Zimbabwe

${ }^{8}$ Division of Infectious Diseases, Department of Medicine, University of Stellenbosch and Tygerberg Academic Hospital, Cape Town, South Africa

${ }^{9}$ Khayelitsha ART Programme, Médecins Sans Frontières, Cape Town, South Africa

${ }^{10}$ Aurum Institute, Johannesburg, South Africa

${ }^{11}$ Johns Hopkins University School of Medicine, Baltimore, MD, USA

${ }^{12}$ Health Economics and Epidemiology Research Office, Department of Internal Medicine, School of Clinical Medicine, Faculty of Health Sciences, University of the Witwatersrand, Johannesburg, South Africa

*Corresponding author e-mail: gwandeler@ispm.unibe.ch

\section{Abstract}

Background: There is debate over using tenofovir or zidovudine alongside lamivudine in second-line antiretroviral therapy (ART) following stavudine failure. We analyzed outcomes in cohorts from South Africa, Zambia and Zimbabwe

Methods: Patients aged $\geq 16$ years who switched from a first-line regimen including stavudine to a ritonavir-boosted lopinavir-based second-line regimen with lamivudine or emtricitabine and zidovudine or tenofovir in seven ART programs in southern Africa were included. We estimated the causal effect of receiving tenofovir or zidovudine on mortality and virological failure using Cox proportional hazards marginal structural models. Its parameters were estimated using inverse probability of treatment weights. Baseline characteristics were age, sex, calendar year and country. CD4 cell count, creatinine and hemoglobin levels were included as time-dependent confounders. 
Results: 1,256 patients on second-line ART, including 958 on tenofovir, were analyzed. Patients on tenofovir were more likely to have switched to second-line ART in recent years, spent more time on first-line ART (33 vs. 24 months) and had lower CD4 cell counts (172 vs. $341 \mathrm{cells} / \mu \mathrm{l})$ at initiation of second-line ART. The adjusted hazard ratio comparing tenofovir with zidovudine was 1.00 (95\% confidence interval 0.59-1.68) for virologic failure and 1.40 (0.57-3.41) for death.

Conclusions: We did not find any difference in treatment outcomes between patients on tenofovir or zidovudine; however, the precision of our estimates was limited. There is an urgent need for randomized trials to inform second-line ART strategies in resource-limited settings.

Accepted 4 November 2013, published online 3 December 2013

Running head: TDF versus AZT in second-line ART

\section{Introduction}

In the absence of routine drug resistance testing in resource-constrained settings, the World Health Organization (WHO) recommends the use of standardized second-line antiretroviral therapy (ART) regimens consisting of a ritonavir-boosted protease inhibitor plus two nucleoside reverse transcriptase inhibitors (NRTI). The NRTI backbone should include lamivudine (or emtricitabine) and tenofovir if stavudine or zidovudine was used in the first-line regimen. However, the K65R mutation, which is associated with tenofovir resistance, is frequent in subtype $C$ viruses, especially in patients on first-line regimens including stavudine [2,3]. Despite these concerns, only few studies have compared clinical outcomes between different second-line regimens in sub-Saharan Africa.

The treatment strategy that should be adopted in case of stavudine failure is of particular importance as most countries are in the process of phasing this drug out [1]. In the absence of virological monitoring in most of these settings, a significant proportion of individuals will have accumulated drug resistance mutations before being switched to other regimens. A recent report on drug resistance patterns in patients failing first-line ART in six African countries suggested that after stavudine failure, zidovudine may be more efficacious than tenofovir [4]. Conversely, a review of HIV-1 resistance mutations data from 35 studies found that tenofovir was more likely than zidovudine to retain antiviral activity following first-line stavudine therapy [5].

We compared clinical outcomes in patients receiving second-line ART including tenofovir or zidovudine after stavudine-failure in a large cohort collaboration in Southern Africa.

\section{Methods}

\section{IeDEA-SA}

The International epidemiological Databases to Evaluate AIDS in Southern Africa (IeDEA-SA) is a collaboration of ART programs in seven countries in Southern Africa [6]. Data are collected at ART 
initiation and each follow-up visit, using standardized instruments, and transferred to data centers at the Universities of Cape Town, Republic of South Africa (RSA), and Bern, Switzerland. All sites have ethical approval to collect data and to participate in leDEA-SA.

\section{Patients and outcomes}

All patients $>16$ years who switched from a first-line regimen including lamivudine, stavudine and either nevirapine or efavirenz to a ritonavir-boosted lopinavir-based second-line regimen with lamivudine or emtricitabine and zidovudine or tenofovir were included. Only cohorts with at least 20 patients meeting the eligibility criteria at the time of the database closure in June 2012 were included. Five programs in South Africa, one in Zambia and one in Zimbabwe met inclusion criteria. The sites in South Africa monitored viral load once or twice a year, whereas the cohorts in Zambia and Zimbabwe mainly relied on immunological and clinical criteria to diagnose treatment failure. The primary outcomes were time to death and, in the South African cohorts, time to virologic rebound or failure. Virologic rebound was defined as a single viral load $>1,000$ copies $/ \mathrm{ml}$, at least six months after the initiation of second-line ART. Virologic failure was defined as a viral load $>1,000$ copies $/ \mathrm{ml}$ after six months confirmed by a second measurement of $>1,000$ copies/ml within one year. Loss to follow-up (LTFU) was defined as not returning to the health-care facility for at least six months.

\section{Statistical analyses}

We used standard crude and adjusted Cox proportional hazards models, adjusting for age, sex, CD4 cell count, hemoglobin and creatinine at start of second-line ART, year of starting second-line ART and country (RSA versus other). Patients were followed-up from the time of initiation of second-line ART to the date the outcome of interest occurred or, if no outcome observed, to the last follow-up visit. We used marginal structural models to adjust for time-dependent confounding by CD4 count, creatinine and hemoglobin level $[7,8]$. A directed acyclic graph (DAG) illustrating the causal structure of the covariates used in the marginal structural models is shown in Supplementary Figure S1. The parameters of these models were estimated using inverse probability of treatment weights [9]. For each semester we used multiple imputation to deal with missing values in CD4 count, creatinine and hemoglobin level. Ten imputed datasets were generated and combined using Rubin's rules [10]. Finally, we examined the crude mean CD4 cell count increase during the first year of second-line ART. All analyses were performed using R-2.14.2 (R Project, Vienna, Austria) and Stata version 12.1 (Stata Corporation, College Station, Texas, USA).

\section{Results}

\section{Baseline characteristics}

Data from 1,256 patients experiencing failure of stavudine-containing first-line ART and switching to second-line ART (tenofovir: 958; zidovudine: 298) were analyzed. Patients receiving tenofovir were older 
(median age: 38 vs. 34 years, $p<0.001)$ and less likely to be female $(64 \%$ vs. $83 \%, p<0.001)$ than those given zidovudine. They were more likely to have switched to second-line ART in recent years, spent more time on first-line ART (median: 33 vs. 24 months, $p<0.001$ ) and had lower CD4 cell counts at initiation of second-line ART (median: 172 vs. 341 cells $/ \mu \mathrm{l}, \mathrm{p}<0.001$ ) compared to patients on zidovudine. Programand patient-level characteristics are described in Table 1 and Supplementary Table S1.

\section{Mortality and treatment failure}

Data up to four years after start of second-line ART were included with a median follow time of 1.23 years in the tenofovir and 1.87 years in the zidovudine group. In all sites combined, 47 patients died and 287 patients were lost over 2,051 person-years of follow-upl. Patients enrolled in South African cohorts had a median of 1.59 viral load measurements per year spent on second-line. In South Africa, 96 patients developed virologic rebound and 26 developed virologic failure. CD4 cell counts were available in $54 \%$ of patients and semesters, values for creatinine in $36 \%$ of patients and levels of hemoglobin in $50 \%$ of patients. The cumulative percentage of patients who were alive and on ART, who died or were lost over four years after starting second-line ART is shown in Figure 1.

In crude analyses rates of virologic rebound and virologic failure were higher with tenofovir than with zidovudine but HRs were compatible with no difference between the two regimens after adjusting for baseline and time-dependent confounders. The adjusted HR from the marginal structural model was 1.30 (95\% Cl 0.94-1.80) for virologic rebound and 1.00 (95\% Cl 0.59-1.68) for virologic failure. Similarly, it was 1.40 (95\% Cl 0.57-3.41) for mortality (Table 2).

Immunological recovery during second-line ART was inferior in patients on tenofovir compared to those on zidovudine (mean increase: 42 vs. 51 CD4 cells/ $\mu$ l), but the difference in CD4 increase between the two treatment groups was small (difference -9 cells, $95 \% \mathrm{Cl}-19$ to $+1, p=0.09$ ).

\section{Discussion}

This is the largest observational study comparing second-line regimens containing tenofovir or zidovudine in sub-Saharan Africa to date. We compared the hazard of virologic rebound, virologic failure, and death, adjusting for confounding by prognostic factors at baseline and for time-dependent confounding by CD4 count, creatinine and hemoglobin. These risk factors vary with time, predict the choice of second-line ART, and are in turn affected by that choice. We found that there was little evidence of a difference in mortality and virologic rebound or failure between patients on second-line ART containing tenofovir or zidovudine after failing a stavudine containing first-line regimen. Similarly there was little difference in immunological response.

The majority of patients failing first-line ART in sub-Saharan Africa have been previously exposed to stavudine. As this drug is being phased out in most countries, many patients are being switched to another NRTI backbone. Studies estimating the residual activity of tenofovir and zidovudine after 
stavudine failure based on drug resistance patterns at first-line failure have been contradictory $[4,5]$. Most studies that evaluated outcomes of second-line ART including tenofovir have shown good treatment responses [11-14]. These findings could, however, be driven by the response to the potent ritonavirboosted protease inhibitor during the first year of second-line ART. Indeed, the two studies showing high rates of re-suppression after first-line failure with proven drug resistances only reported on 12-month outcomes [12,14]. Our study extends these results by showing that outcomes in patients on tenofovircontaining second-line ART are favorable and similar to those observed with zidovudine for up to four years after initiating second-line ART.

The number of outcomes observed in our study was relatively small and statistical power to detect smaller differences between the two regimens was therefore limited. Also, there were important imbalances in baseline characteristics. For instance, patients on tenofovir spent more time on their firstline regimen: they may have developed drug resistance mutations limiting the effectiveness of second-line ART. Patients given tenofovir also had lower CD4 cell counts at initiation of second-line ART than those given zidovudine. Finally, missing values were a problem. We imputed these data and adjusted for baseline and time-dependent confounding; however, we recognize that residual confounding may have affected our results. As we had little data on drug resistance patterns, we were unable to assess the impact of specific resistance mutations on treatment outcomes. However, drug resistance is only one of multiple important drivers of treatment failure. Incomplete adherence may be an even stronger risk factor for treatment failure in patients on second-line ART.

In conclusion, we did not detect important differences in virologic or immunologic response or mortality in patients who failed a stavudine containing first-line regimen and switched to second-line ART including tenofovir or zidovudine. The precision of our estimates was, however, limited and there is clearly a need for continued monitoring of outcomes of second-line ART in sub-Saharan Africa, within leDEA and other cohort collaborations. Although our results are reassuring, randomized trials would provide stronger evidence regarding optimal choices of second-line ART in Southern Africa.

\section{Acknowledgements}

We thank all study participants and staff of all participating sites. We also thank Michael Shomaker for his valuable statistical inputs

\section{Author contribution}

GW and ME designed the study and wrote the first draft of the manuscript. FG, MZ and TG conducted the statistical analyses. All authors contributed to the interpretation of the data, critically revised the paper and approved its final version.

\section{Financial support}

This study was supported by the National Institute of Allergy and Infectious Diseases (grant 1 U01AI069924-01). The funders had no role in study design, data collection and analysis, decision to publish, or preparation of the manuscript. Matthew Fox was supported by the National Institute of Allergy and Infectious Diseases [K01AI083097]. Julia Rohr was supported by Cooperative Agreement AID 674-A12-00029 from the United States Agency for International Development (USAID). The contents are the 
responsibility of the authors and do not necessarily reflect the views of NIH, USAID or the United States Government.

\section{Potential conflicts of interests}

We declare no competing interests.

\section{References}

1. World Health Organization. Antiretroviral therapy for HIV infection in adults and adolescents: recommendations for a public health approach. 2010 revision. Geneva: World Health Organization; 2010. Available at http://www.who.int/hiv/pub/arv/adult2010/en/index.html.

2. Brenner BG, Coutsinos D. The K65R mutation in HIV-1 reverse transcriptase: genetic barriers, resistance profile and clinical implications. HIV Ther. 2009;3 [6]:583-94. Epub 2010/03/02.

3. Hosseinipour MC, van Oosterhout JJ, Weigel R, Phiri S, Kamwendo D, Parkin N, et al. The public health approach to identify antiretroviral therapy failure: high-level nucleoside reverse transcriptase inhibitor resistance among Malawians failing first-line antiretroviral therapy. AIDS. 2009;23 [9]:1127-34. Epub 2009/05/07.

4. Hamers RL, Sigaloff KC, Wensing AM, Wallis CL, Kityo C, Siwale M, et al. Patterns of HIV-1 Drug Resistance After First-Line Antiretroviral Therapy (ART) Failure in 6 Sub-Saharan African Countries: Implications for Second-Line ART Strategies. Clin Infect Dis. 2012;54 [11]:1660-9. Epub 2012/04/05.

5. Tang MW, Rhee SY, Bertagnolio S, Ford N, Holmes S, Sigaloff KC, et al. Nucleoside reverse transcriptase inhibitor resistance mutations associated with first-line Stavudine-containing antiretroviral therapy: programmatic implications for countries phasing out Stavudine. J Infect Dis. 2013;207 Suppl 2:S70-7. Epub 2013/05/25.

6. Egger M, Ekouevi DK, Williams C, Lyamuya RE, Mukumbi H, Braitstein P, et al. Cohort Profile: The international epidemiological databases to evaluate AIDS (leDEA) in sub-Saharan Africa. Int J Epidemiol. 2011. Epub 2011/05/20

7. Robins JM, Hernan MA, Brumback B. Marginal structural models and causal inference in epidemiology. Epidemiology. 2000;11 [5]:550-60. Epub 2000/08/24.

8. Pearl J. An introduction to causal inference. The international journal of biostatistics. 2010;6 [2]:Article 7. Epub 2010/03/23.

9. Cole SR, Hernan MA. Constructing inverse probability weights for marginal structural models. American journal of epidemiology. 2008;168 [6]:656-64. Epub 2008/08/07.

10. D. R. Multiple imputation for nonresponse in surveys. New York: Wiley. 1987.

11. Murphy RA, Sunpath H, Lu Z, Chelin N, Losina E, Gordon M, et al. Outcomes after virologic failure of first-line ART in South Africa. AIDS. 2010;24 [7]:1007-12. Epub 2010/04/17.

12. Sigaloff KC, Hamers RL, Wallis CL, Kityo C, Siwale M, Ive P, et al. Second-Line Antiretroviral Treatment Successfully Resuppresses Drug-Resistant HIV-1 After First-Line Failure: Prospective Cohort in Sub-Saharan Africa. J Infect Dis. 2012;205 [11]:1739-44. Epub 2012/03/27.

13. Wandeler G, Keiser O, Mulenga L, Hoffmann CJ, Wood R, Chaweza T, et al. Tenofovir in second-line ART in Zambia and South Africa: collaborative analysis of cohort studies. J Acquir Immune Defic Syndr. 2012;61 [1]:41-8. Epub 2012/06/30.

14. Hosseinipour MC, Kumwenda JJ, Weigel R, Brown LB, Mzinganjira D, Mhango B, et al. Second-line treatment in the Malawi antiretroviral programme: high early mortality, but good outcomes in survivors, despite extensive drug resistance at baseline. HIV Med. 2010;11 [8]:510-8. Epub 2010/03/30. 
Publication: Antiviral Therapy; Type: Short communication

DOI: $10.3851 / \mathrm{IMP} 2710$

Table 1: Comparison of patients at the initiation of second-line ART, by treatment regimen.

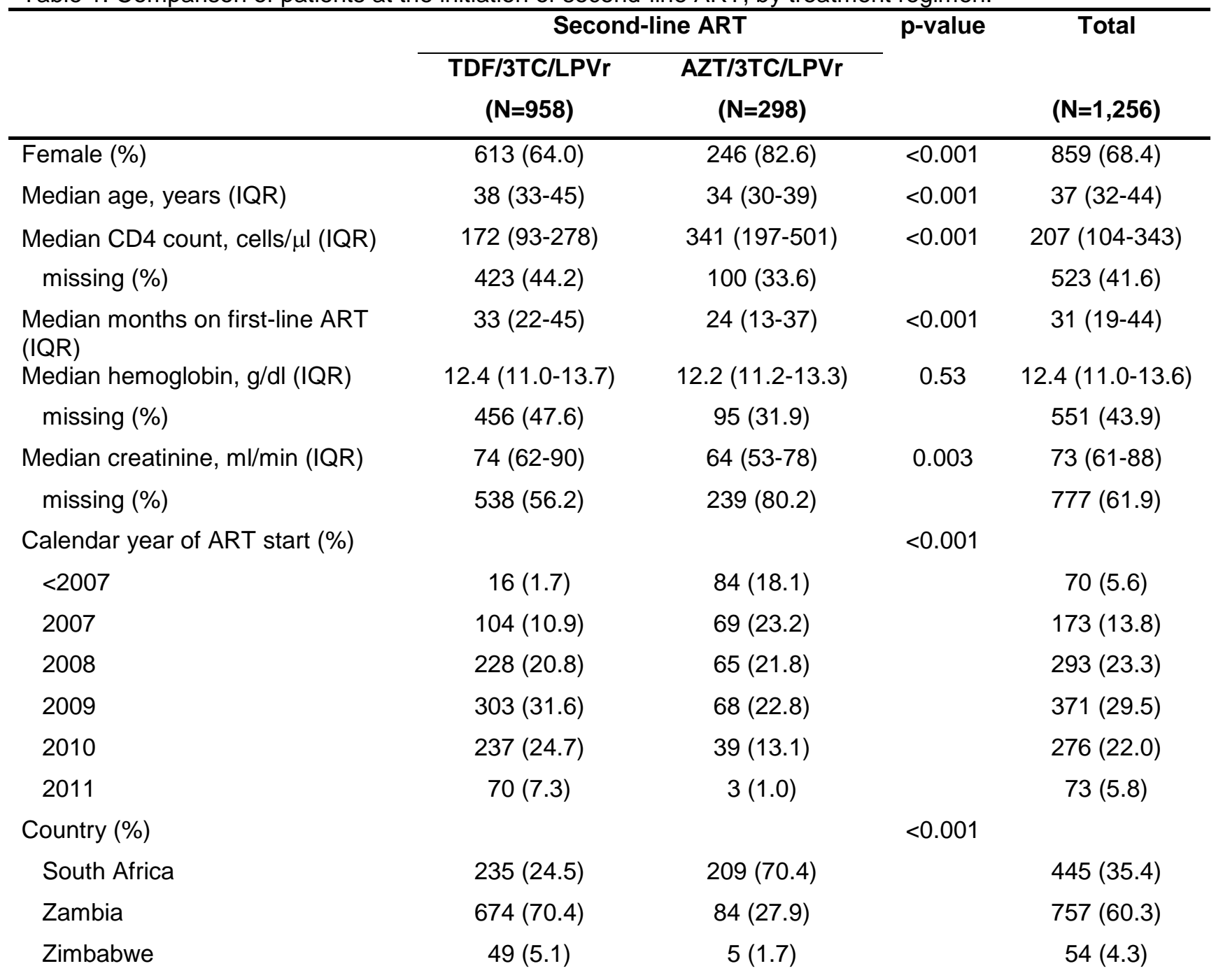

ART, antiretroviral treatment; TDF, tenofovir; 3TC: lamivudine, LPVr: lopinavir-ritonavir, AZT: zidovudine, IQR, interquartile range, 
Publication: Antiviral Therapy; Type: Short communication

DOI: $10.3851 / \mathrm{IMP} 2710$

Table 2: Mortality, virologic rebound and virologic failure in patients on tenofovir or zidovudine containing second-line regimen after failing a stavudine containing first-line regimen in Southern Africa.

No. of events

(\%)
Incidence rate
(per 100 py) Unadjusted hazard
ratio from Cox model $(95 \% \mathrm{Cl})$

el Adid

Adjusted hazard ratio from standard Cox model $(95 \% \mathrm{Cl})$ AZT

\section{Virologic rebound}

Virologic failure

\section{Mortality}

$\begin{array}{cc}\text { TDF } & \text { AZT } \\ 55(23.40) & 41(19.50)\end{array}$

TDF

AZT

* Adjusted for a

$11(4.68) \quad 15(7.14)$

23.60

4.73

8.28

3.03

2.60

1.65

$1.96(1.53-2.50)$
$2.21(1.53-3.21)$
$1.70(0.83-3.51)$

$1.30(0.94-1.79)$
$1.04(0.63-1.73)$
$1.39(0.57-3.39)$

1.65

TDF: tenofovir, AZT: zidovudine, $95 \% \mathrm{Cl}$ : $95 \%$ Confidence Interval, py: person years

Virological rebound: viral load $>1,000 \mathrm{copies} / \mathrm{ml}$ after at least 6 months of follow-up

Virological failure: 2 consecutive viral loads $>1,000$ copies $/ \mathrm{ml}$ after at least 6 months of follow-up 
Supplementary Table S1: Characteristics of patients included in an analysis comparing zidovudine with tenofovir containing second-line antiretroviral therapy in Southern Africa.

\begin{tabular}{|c|c|c|c|c|c|c|c|}
\hline \multirow[t]{2}{*}{ Sites } & \multirow{2}{*}{$\begin{array}{c}\text { No. of } \\
\text { patients }\end{array}$} & \multicolumn{2}{|c|}{ Second-line ART } & \multirow[t]{2}{*}{ Female (\%) } & \multirow{2}{*}{$\begin{array}{c}\text { Median age in } \\
\text { years (IQR) }\end{array}$} & \multirow{2}{*}{$\begin{array}{l}\text { Median follow- } \\
\text { up time (days) }\end{array}$} & \multirow{2}{*}{$\begin{array}{l}\text { Median number } \\
\text { of VL per year } \\
\text { on SL (IQR) }\end{array}$} \\
\hline & & TDF/3TC/LPVr & AZT/3TC/LPVr & & & & \\
\hline \multicolumn{8}{|l|}{ South Africa } \\
\hline Aurum CS & 33 & $21(63.6)$ & $12(36.4)$ & $20(60.6)$ & $39(33-44)$ & $405(267-741)$ & $2.29(2.03-2.75)$ \\
\hline Gugulethu & 88 & $34(38.6)$ & $54(61.4)$ & $70(79.6)$ & $34(31-38)$ & $622(435-1,592)$ & $2.86(2.41-3.18)$ \\
\hline Khayelitsha & 186 & $146(78.5)$ & $40(21.5)$ & $129(69.4)$ & $35(31-39)$ & $355(197-491)$ & $0(0-0)$ \\
\hline Themba Lethu & 115 & $24(20.9)$ & $91(79.1)$ & $100(87.0)$ & $35(30-38)$ & $569(317-920)$ & $2.31(1.67-3.17)$ \\
\hline Tygerberg & 23 & $10(43.5)$ & $13(56.5)$ & $19(82.6)$ & $33(27-39)$ & $246(80-379)$ & $1.32(0-4.11)$ \\
\hline \multicolumn{8}{|l|}{ Zambia } \\
\hline MOH-CIDRZ & 757 & $674(89.0)$ & $83(11.0)$ & $479(63.3)$ & $39(33-45)$ & $531(304-802)$ & NA \\
\hline \multicolumn{8}{|l|}{ Zimbabwe } \\
\hline Newlands & 54 & $49(90.7)$ & $5(9.3)$ & $42(77.8)$ & $40(33-45)$ & $735(357-1,226)$ & NA \\
\hline Total & 1,256 & $958(76.3)$ & $298(23.7)$ & $43,654(60.1)$ & $37(32-44)$ & $489(292-826)$ & $1.59(0-2.78)$ \\
\hline
\end{tabular}

TDF: tenofovir, 3TC: lamivudine, LPVr: lopinavir-ritonavir, AZT: zidovudine, CS: community site, CIDRZ: Centre for

Infectious Disease Research in Zambia, IQR: inter-quartile range; VL: viral load; SL: second-line antiretroviral therapy; NA: not applicable 
Supplementary Figure S1: Directed acyclic graph (DAG) illustrating the causal structure of the covariates used in the marginal structural model of the effect of zidovudine vs. tenofovir in second-line ART on mortality.

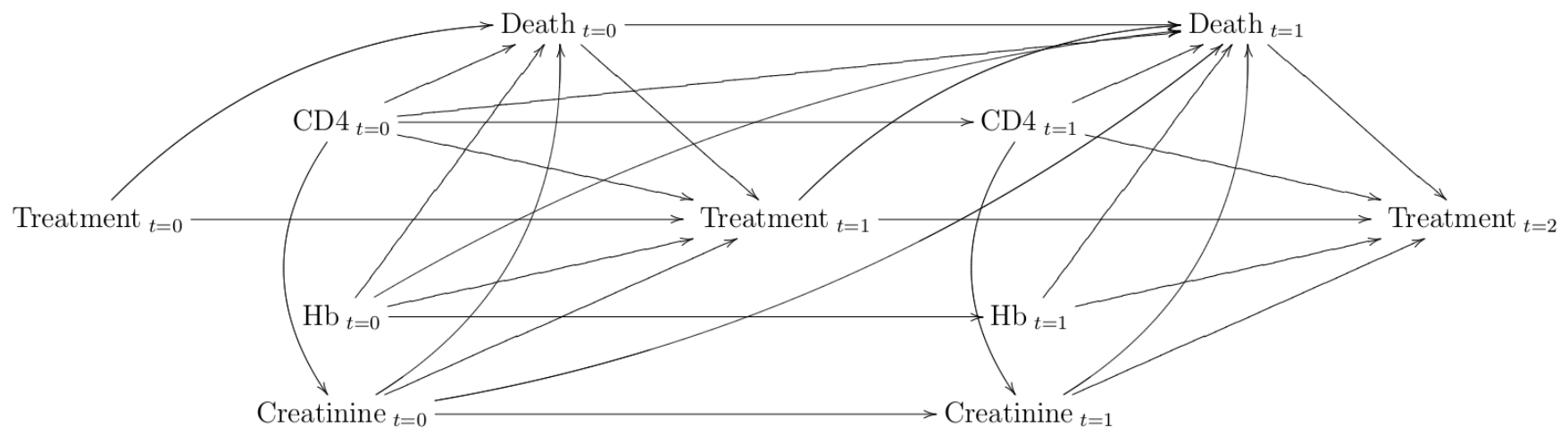

* Hb: hemoglobin 